\title{
Enhanced spontaneous emission rate in annular plasmonic nanocavities
}

\author{
E. J. A. Kroekenstoel, E. Verhagen, ${ }^{a)}$ R. J. Walters, L. Kuipers, and A. Polman \\ Center for Nanophotonics, FOM Institute AMOLF, Science Park 104, 1098 XG Amsterdam, The Netherlands
}

(Received 7 September 2009; accepted 2 December 2009; published online 31 December 2009)

\begin{abstract}
The spontaneous emission rate of erbium ions is enhanced by coupling to localized plasmonic resonances in subwavelength annular apertures in a $\mathrm{Au}$ film. The $\mathrm{Er}^{3+}$ ions, embedded in $\mathrm{SiO}_{2}$, are selectively located inside the apertures. The annular apertures act as nanocavities, enhancing the local density of optical states at the Er emission wavelength of $1.54 \mu \mathrm{m}$ when the cavities are tuned to that wavelength. We show that this leads to an eightfold increase of the photoluminescence intensity, in conjunction with a 2.4-fold enhancement of the spontaneous emission rate. (c) 2009 American Institute of Physics. [doi:10.1063/1.3276566]
\end{abstract}

Control of spontaneous emission is of interest for many applications, including LEDs, sensors, quantum information processing, and photovoltaics. ${ }^{1}$ By modifying the local electromagnetic environment of an optical emitter, the polarization and the directionality of its radiation as well as its overall decay rate can be modified. In recent years a number of different plasmonic geometries have been used to modify the spontaneous emission rate, including nanoparticles and nanoantennas. ${ }^{2-7}$

Suitably shaped subwavelength apertures in a metal film can also support a localized plasmonic resonance. For example, annular apertures can act as nanocavities with a modal field that is strongly confined to the aperture. ${ }^{8,9}$ Even though the quality factor $Q$ of these cavities is modest, the ultrasmall mode volume $V$ can potentially lead to a significant Purcell enhancement of the spontaneous emission rate, which is given by $F=3 \lambda_{0}^{3} Q /\left(4 \pi^{2} V\right)$, where $\lambda_{0}$ is the freespace wavelength. ${ }^{10}$ Because of the relatively low $Q$, any Purcell enhancement induced by the localized plasmon resonances would be observed over a large bandwidth. It is well known that annular apertures couple strongly to far-field radiation, since they can assist transmission of light through a metal film. ${ }^{8,9}$ This makes these structures promising candidates for enhancing the radiative emission rate. Previous studies of fluorescence from circular subwavelength apertures have demonstrated the modification of spontaneous emission in various ways, ${ }^{11-14}$ but these did not exploit localized resonances in the apertures.

In this work, we demonstrate the enhancement of the radiative emission rate of optically active erbium ions ${ }^{15}$ positioned inside annular apertures in a gold film. We observe an increase in both PL intensity and decay rate as we tune the apertures' resonance wavelength to the Er transition wavelength.

To obtain maximum spatial overlap of the Er ions with the nanocavity modal field the ions have to be positioned inside the annular apertures. A schematic of the fabricated structures is shown in Fig. 1(a). A fused silica (Heraeus Suprasil 300) substrate is first doped with erbium by $150 \mathrm{keV}$ $\mathrm{Er}^{+}$ion implantation at normal incidence using a fluence of $3.0 \times 10^{14} \mathrm{~cm}^{-2}$. This results in a distribution of Er ions at a mean depth of $82 \mathrm{~nm}$ with a standard deviation of $18 \mathrm{~nm}$ and a peak concentration of 0.29 at. \%, as calculated with the

${ }^{a)}$ Electronic mail: verhagen@amolf.nl.
Monte Carlo program SRIM. ${ }^{16}$ We then remove the top 30 $\mathrm{nm}$ of silica with reactive ion etching in order to reduce the mean depth of the Er ions to $50 \mathrm{~nm}$. Next, the substrate is covered with a resist stack of $500 \mathrm{~nm}$ PMMA, $20 \mathrm{~nm}$ Ge and $90 \mathrm{~nm}$ negative electron beam resist (Ma-N 2401) in which we pattern $50 \times 50 \mu \mathrm{m}^{2}$ hexagonally ordered arrays of annular structures by electron beam lithography. Square regions near these arrays are also exposed to provide reference areas. After developing the patterned layer, reactive ion etching through the resist layers and through $100 \mathrm{~nm}$ of silica is used to leave $100 \mathrm{~nm}$ thick Er-doped silica rings on the substrate. A $100 \mathrm{~nm}$ thick layer of gold is evaporated on the sample and the excess gold and resist is removed using lift-off. This results in annular apertures in a $\mathrm{Au}$ film on $\mathrm{SiO}_{2}$, filled with Er-doped $\mathrm{SiO}_{2}$. A top-view SEM image of part of an array is depicted in Fig. 1(b). A small air gap is observed between the $\mathrm{SiO}_{2}$ rings and surrounding $\mathrm{Au}$.

Optical transmission spectra of the arrays are obtained by illuminating the sample from the air side with light from an incandescent lamp (tungsten-halogen) with a numerical aperture (NA) of 0.02 and collecting the transmitted light through a $0.75 \mathrm{NA}$ objective. The light is passed through a spectrometer and detected with either a silicon CCD for visible wavelengths or an InGaAs diode array for infrared light. Reflection spectra are recorded using a 0.65 NA reflective objective and a Biorad FTS6000 Fourier transform spectrometer with an InAs photodiode.

To measure the photoluminescence (PL) of the erbium ions the sample is illuminated with light from an argon ion laser operating at $488 \mathrm{~nm}$ incident under a $20^{\circ}$ angle to the sample surface from the air side of the metal film. The light is focused to an elliptically shaped Gaussian spot with a full
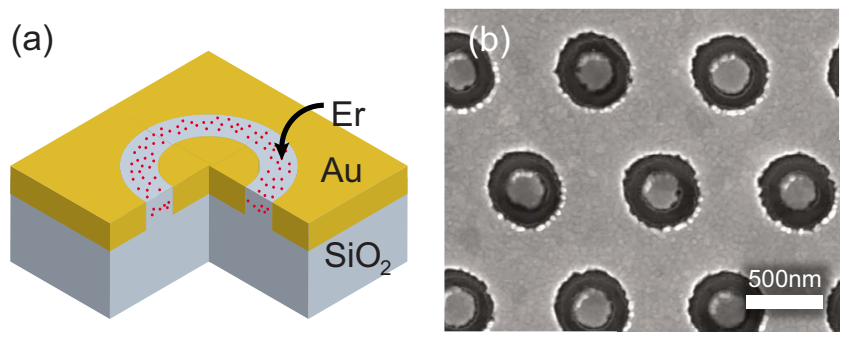

FIG. 1. (Color online) (a) Schematic of an Er-doped $\mathrm{SiO}_{2}$ annular aperture in a $\mathrm{Au}$ film on $\mathrm{SiO}_{2}$. (b) SEM image of a fabricated hexagonal array of annular apertures. 


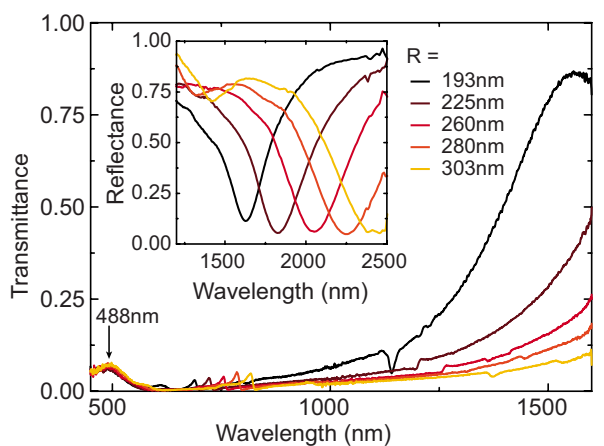

FIG. 2. (Color online) Transmission and reflection (inset) spectra for the annular aperture arrays. By increasing the average radius of the annular apertures the resonance wavelength is shifted. The pump wavelength $(488 \mathrm{~nm})$ in PL experiments is indicated by an arrow.

width at half maximum of $34 \mu \mathrm{m}$ along one direction and $85 \mu \mathrm{m}$ along the other. The peak power density is $275 \mathrm{~W} / \mathrm{cm}^{2}$ for intensity measurements and $680 \mathrm{~W} / \mathrm{cm}^{2}$ for time-resolved measurements. We collect the luminescence with a $0.45 \mathrm{NA}$ objective at the air side of the sample. PL intensity is measured with a spectrometer with an InGaAs diode array detector and decay rate measurements are performed with a North Coast EO-817s Ge photodiode detector with a response time of $30 \mu$ s while the laser is modulated at $7 \mathrm{~Hz}$ with an acousto-optic modulator.

The resonance wavelength of the annular aperture nanocavities can be tuned by varying the aperture size and shape. The localized resonance that is considered here can be related to the $\mathrm{TE}_{11}$ mode of a coaxial waveguide with an equivalent cross section. ${ }^{8,9}$ At cutoff the wavevector of this mode becomes close to zero, resulting in resonant constructive interference inside the aperture for arbitrary film thickness. The cutoff wavelength for the $\mathrm{TE}_{11}$ mode in a coaxial perfect conductor waveguide is approximated by $n\left(R_{\mathrm{O}}\right.$ $\left.+R_{\mathrm{i}}\right) \pi,{ }^{8,9}$ where $R_{\mathrm{o}}, R_{\mathrm{i}}$, and $n$ are the outer and inner radius of the aperture and the refractive index of the medium in the waveguide, respectively. We control the resonance wavelength by varying the average radius $R=\left(R_{\mathrm{O}}+R_{\mathrm{i}}\right) / 2$ from 193 to $303 \mathrm{~nm}$. The width, $R_{\mathrm{o}}-R_{\mathrm{i}}$, is kept constant at $100 \mathrm{~nm}$ to maintain a constant average separation between Er ions and the metal surface, and therefore a constant rate of direct quenching to the metal. ${ }^{17}$ Concurrent with the increase in $R$ the periodicity is increased from 903 to $1086 \mathrm{~nm}$ such that the average areal density of Er ions is the same in all arrays.

Transmission and reflection spectra of the arrays with varying $R$ are shown in Fig. 2. A large transmittance peak is seen in conjunction with a dip in reflectance, associated with the localized resonance. As expected, the resonance wavelength shifts to larger wavelengths when $R$ is increased, varying from $\sim 1.6$ to $2.4 \mu \mathrm{m}$. In Fig. 3(a) we plot the transmittance at $1.54 \mu \mathrm{m}$ for the different cavities as a function of $R$ : it reduces from 0.84 to 0.08 . As all transmission at this wavelength is mediated by the localized resonance, the transmittance can be used as a measure of the strength of the resonant mode at a given frequency. The decreasing transmittance for increasing $R$ therefore implies that the local density of optical states (LDOS) at the Er transition wavelength will decrease as the radius is increased.

A typical PL spectrum of the $\mathrm{Er}^{3+}{ }^{4} \mathrm{I}_{13 / 2} \rightarrow{ }^{4} \mathrm{I}_{15 / 2}$ transition at $1.54 \mu \mathrm{m}$ is shown in Fig. 4(a). We compare the integrated PL intensity in Au annular nanocavities to that in

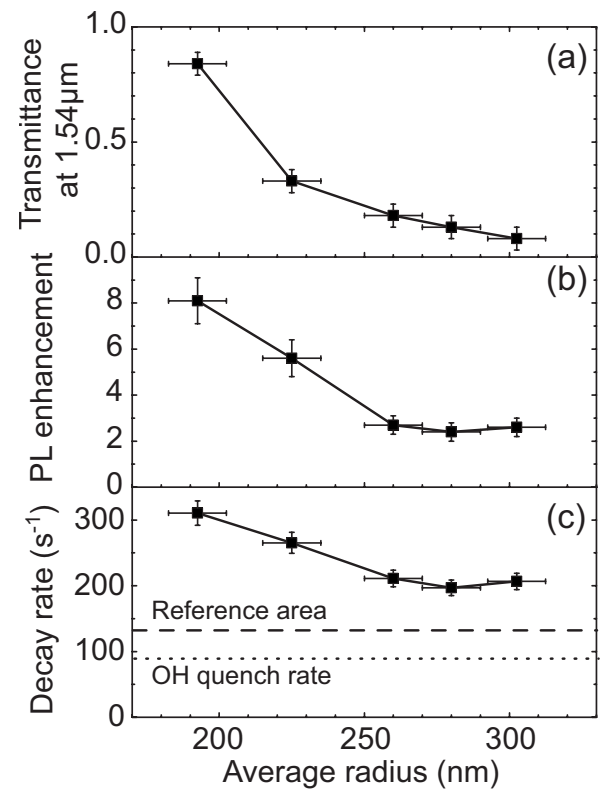

FIG. 3. Optical properties of Er-doped annular apertures as a function of average aperture radius $R$. (a) Transmittance at $1.54 \mu \mathrm{m}$; (b) PL enhancement compared to similar arrays of silica rings not covered in $\mathrm{Au}$; (c) $\mathrm{Er}$ decay rate at $1.54 \mu \mathrm{m}$. The decay rate of $\mathrm{Er}$ in a reference area is indicated by a dashed line and the rate due to $\mathrm{OH}$ quenching by the dotted line.

similar arrays of silica rings that are not covered in $\mathrm{Au}$. In Fig. 3(b) we plot the PL enhancement, taken as the ratio of the PL intensity from the $\mathrm{Au}$ nanocavities to that from the silica rings without $\mathrm{Au}$. We observe an enhancement up to a factor 8 for the annular apertures with a resonance near the erbium emission wavelength, i.e., for the apertures with $R$ $=193 \mathrm{~nm}$. As we increase $R$ the resonance wavelength in creases, shifting away from the Er emission wavelength [see Fig. 3(a)], and the PL enhancement decreases, demonstrating a strong correlation between the PL enhancement and cavity resonance condition.

To investigate the origin of the observed trend in the PL enhancement with $R$, we performed time-resolved measurements of the PL decay. For the apertures with a resonance near $1.6 \mu \mathrm{m}(R=193 \mathrm{~nm})$ and the ones with a resonance the furthest away from the erbium emission wavelength $(R$ $=303 \mathrm{~nm}$ ) the decay curves are plotted in Fig. 4(b). Singleexponential functions are fitted to the curves to obtain a first-order estimate of the decay rate of Er in the various nanocavities. The fitted decay rates for all arrays are plotted
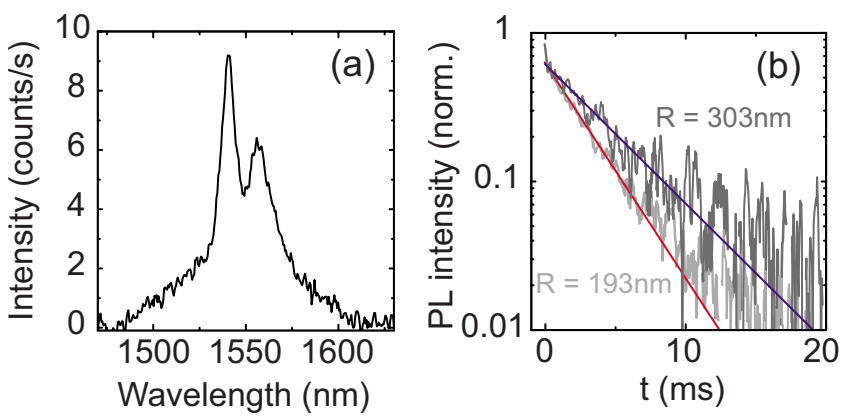

FIG. 4. (Color online) (a) Erbium luminescence spectrum taken on a planar $\mathrm{SiO}_{2}$ film $\left(\lambda_{\text {pump }}=488 \mathrm{~nm}\right)$ and (b) decay curves for two different annular aperture arrays with a resonance near the erbium emission wavelength $(R=193 \mathrm{~nm})$ and with a resonance the furthest away from $1.5 \mu \mathrm{m}$ $(R=303 \mathrm{~nm})$. 
in Fig. 3(c). We find that the total decay rate for the resonant array with $R=193 \mathrm{~nm}\left(\Gamma=311 \mathrm{~s}^{-1}, \tau=3.2 \mathrm{~ms}\right)$ is 1.5 times as large as that for apertures with $R=303 \mathrm{~nm}$. $\left(\Gamma=207 \mathrm{~s}^{-1}, \quad \tau=4.8 \mathrm{~ms}\right)$ The decay rate for $\mathrm{Er}$ in the unpatterned reference area without $\mathrm{Au}$ is $\Gamma=132 \mathrm{~s}^{-1}(\tau=7.6 \mathrm{~ms})$, shown as the dashed line in Fig. 3(c). Accordingly, the total decay rate of Er for the resonant apertures is increased by a factor 2.4 as compared to that of Er in the reference area.

The corresponding trends in PL intensity and decay rate with varying $R$ are a strong indication that the observed increase of the PL intensity as the resonance is tuned to the Er transition is related to an increase of the radiative decay rate. As the PL experiments were performed in the linear pumping regime, the increase in PL enhancement from 2.5 for apertures with large radii to 8.1 for the apertures with $R$ $=193 \mathrm{~nm}$ indicates that the luminescence quantum efficiency is increased by a factor 3 as we tune the resonance wavelength to the Er emission wavelength. We exclude a difference in pump rate for the various aperture arrays as an alternative cause for the trend observed in the PL intensity. The pump wavelength is much smaller than the resonance wavelength and we observe no change in the transmission near $488 \mathrm{~nm}$ for the various aperture arrays. Although it is in principle possible that the pump intensity is overall enhanced by an approximately equal amount in all structures, this could neither explain the observed trend of PL intensity with varying $R$ nor the change of the decay rate.

It is possible to estimate an upper bound to the enhancement of the radiative rate when the structure is tuned to resonance. The total decay rate $\Gamma$ is composed of radiative and nonradiative components. There are two sources for nonradiative decay: quenching by $\mathrm{OH}$ impurities in the glass, ${ }^{15}$ which is independent of the dielectric environment, ${ }^{18}$ and absorption by the Au. The $\mathrm{OH}$ quench rate, derived from a comparison of the measured decay rate of $\mathrm{Er}$ in the reference area, the known radiative decay rate in bulk $\mathrm{SiO}_{2}\left(54 \mathrm{~s}^{-1}\right),{ }^{19}$ and the known relative LDOS at the depth of the Er ions in the reference area, ${ }^{20}$ amounts to $\Gamma_{\mathrm{OH}}=89 \mathrm{~s}^{-1}$ [dotted line in Fig. 3(c)]. Correcting for the LDOS-independent $\mathrm{OH}$ quenching, the remaining decay rate, i.e., $\Gamma-\Gamma_{\mathrm{OH}}$, for the apertures resonant near the Er transition wavelength is increased by a factor 4.1 as compared to the radiative decay rate in bulk $\mathrm{SiO}_{2}$. Taking into account the refractive index of $\mathrm{SiO}_{2}$, the remaining decay rate is thus enhanced by a factor 6 with respect to the decay rate in vacuum. This number constitutes an upper limit to the enhancement of the radiative rate, if the absorption by $\mathrm{Au}$ is negligible compared to the power radiated to the far field by the aperture mode.

The quality factor derived from the reflectance data is $Q=5.2$ for the array with $R=193 \mathrm{~nm}$. A first estimate of the expected Purcell factor $F$ of 135 is obtained by taking the mode volume equal to the geometrical volume (1.3 $\times 10^{-2} \mu \mathrm{m}^{3}$ ). This estimate of the maximum enhancement of the decay rate of an optimally positioned and oriented dipole emitter is much larger than the experimentally derived rate enhancement of a factor 6 . The difference suggests that the coupling of Er to the nanocavity mode is less than opti- mal. The fact that only one particular emission dipole orientation will couple efficiently to the plasmon mode can account for a reduction of the rate enhancement by a factor $\sim 3$. The difference can be furthermore due to a possible spatial mismatch between the Er distribution and the $\mathrm{TE}_{11}$ mode profile and the slight detuning of the resonance frequency to the emission wavelength. Moreover, the effective mode volume could differ significantly from the geometrical volume. ${ }^{21,22}$

In summary, we have shown that the spontaneous emission decay rate of $\mathrm{Er}^{3+}$ ions at $1.54 \mu \mathrm{m}$ is controlled using annular apertures in a Au film. A 2.4-fold decay rate enhancement and a eightfold PL intensity enhancement are observed for apertures filled with Er-doped $\mathrm{SiO}_{2}$ when the localized plasmon resonance of the apertures is tuned to the $\mathrm{Er}$ emission wavelength.

The authors thank Chris Rétif, Simon Huisman, and Willem Vos for technical assistance and the use of equipment. This work is part of the Joint Solar Programme (JSP) of FOM, which is financially supported by NWO. The JSP is cofinanced by gebied Chemische Wetenschappen of NWO and Stichting Shell Research.

${ }^{1}$ P. Bharadwaj, B. Deutsch, and L. Novotny, Adv. Opt. Photon. 1, 438 (2009).

${ }^{2}$ J. N. Farahani, D. W. Pohl, H.-J. Eisler, and B. Hecht, Phys. Rev. Lett. 95, 017402 (2005).

${ }^{3}$ I. Gryczynski, J. Malicka, E. Holder, N. DiCesare, and J. R. Lakowicz, Chem. Phys. Lett. 372, 409 (2003).

${ }^{4}$ H. Mertens, J. S. Biteen, H. A. Atwater, and A. Polman, Nano Lett. 6, 2622 (2006).

${ }^{5}$ S. Kühn, U. Håkanson, L. Rogobete, and V. Sandoghdar, Phys. Rev. Lett. 97, 017402 (2006).

${ }^{6}$ F. Tam, G. P. Goodrich, B. R. Johnson, and N. J. Halas, Nano Lett. 7, 496 (2007).

${ }^{7}$ O. L. Muskens, V. Giannini, J. A. Sanchez-Gil, and J. Gomez Rivas, Nano Lett. 7, 2871 (2007).

${ }^{8}$ F. Baida, D. Van Labeke, G. Granet, A. Moreau, and A. Belkhir, Appl. Phys. B: Lasers Opt. 79, 1 (2004).

${ }^{9}$ W. Fan, S. Zhang, B. Minhas, K. J. Malloy, and S. R. J. Brueck, Phys. Rev. Lett. 94, 033902 (2005).

${ }^{10}$ E. M. Purcell, Phys. Rev. 69, 681 (1946).

${ }^{11}$ Y. Liu and S. Blair, Opt. Lett. 28, 507 (2003).

${ }^{12}$ S. H. Garrett, L. H. Smith, and W. L. Barnes, J. Mod. Opt. 52, 1105 (2005).

${ }^{13}$ A. G. Brolo, S. C. Kwok, M. G. Moffitt, R. Gordon, J. Riordon, and K. L. Kavanagh, J. Am. Chem. Soc. 127, 14936 (2005).

${ }^{14}$ J. Wenger, D. Gérard, J. Dintinger, O. Mahboub, N. Bonod, E. Popov, T. W. Ebbesen, and H. Rigneault, Opt. Express 16, 3008 (2008).

${ }^{15}$ A. Polman, J. Appl. Phys. 82, 1 (1997).

${ }^{16}$ www.srim.org

${ }^{17}$ J. Kalkman, L. Kuipers, A. Polman, and H. Gersen, Appl. Phys. Lett. 86, 041113 (2005).

${ }^{18}$ M. J. A. de Dood, J. Knoester, A. Tip, and A. Polman, Phys. Rev. B 71, 115102 (2005).

${ }^{19}$ M. J. A. de Dood, L. H. Slooff, A. Polman, A. Moroz, and A. van Blaaderen, Phys. Rev. A 64, 033807 (2001).

${ }^{20}$ E. Snoeks, A. Lagendijk, and A. Polman, Phys. Rev. Lett. 74, 2459 (1995).

${ }^{21}$ S. Maier, Opt. Quantum Electron. 38, 257 (2006).

${ }^{22}$ Y. C. Jun, R. D. Kekatpure, J. S. White, and M. L. Brongersma, Phys. Rev. B 78, 153111 (2008). 